\title{
Informação Especialista para um Sistema de Filtragem Online com Ensemble de Redes Neurais em um Ambiente com Alta Taxa de Eventos
}

\author{
Edmar E. P. de Souza ${ }^{1}$, Juan L. Marin ${ }^{1}$, Eduardo F. S. Filho ${ }^{1}$, Paulo C. M. A. Farias ${ }^{1}$, \\ João V. F. Pinto ${ }^{2}$, Micael V. de Araújo ${ }^{2}$, José M. de Seixas ${ }^{2}$ \\ ${ }^{1}$ Laboratório de Sistemas Digitais, PPGEE/UFBA \\ ${ }^{2}$ Laboratório de Processamento de Sinais, COPPE/POLI,UFRJ \\ Emails: \{edmar.egidio, juan.lieber, eduardo.simas, paulo.farias\}@ufba.br, \\ \{jodafons,micael.verissimo, seixas\}@lps.ufrj.br
}

\begin{abstract}
Resumo-O experimento ATLAS é projetado para observar os constituintes fundamentais em colisões de partículas no Grande Colisor de Hádrons (LHC). Na tomada de dados a partir de 2021, espera-se aumentar a energia de colisão e a taxa de produção de informações, o que requer melhorias no sistema de filtragem online. $\mathrm{O}$ NeuralRinger é o algoritmo padrão de identificação online de elétrons no ATLAS (etapa rápida) e usa informações do calorímetro (medição de energia) organizadas em anéis concêntricos para alimentar um conjunto de redes neurais. Uma das principais características utilizadas para a discriminação de elétrons e jatos hadrônicos é o perfil lateral do chuveiro de partículas de energia decrescente medido no calorímetro, pois o chuveiro desenvolvido por partículas eletromagnéticas tende a ser menos largo lateralmente se comparado a chuveiros hadrônicos. Neste trabalho, é investigada a utilização do perfil lateral do chuveiro combinado com os anéis. $O$ método proposto pondera os anéis gerados pelo NeuralRinger pela energia e distância até a célula mais energética. Os resultados indicaram uma redução do falso alarme de 1,37 pontos percentuais quando comparado ao desempenho do NeuralRinger em dados experimentais o que é relevante, haja vista a alta taxa de eventos gerados no ATLAS.
\end{abstract}

Palavras-chave - Redes Neurais, NeuralRinger, Física de Altas Energias, Identificação de elétrons

\section{INTRODUÇÃO}

Experimentos modernos de física de altas energias (High Energy Physics - HEP) requerem notável infraestrutura tecnológica para observação de partículas e interações com rara ocorrência [1] [2]. Em geral, durante a tomada de dados a maior parte da informação coletada já é conhecida (ruído de fundo), exigindo sistemas eficientes de filtragem online para preservar o evento de interesse (sinal) e rejeitar o ruído de fundo [3]. A filtragem de eventos em HEP é uma tarefa desafiadora porque uma enorme quantidade de dados é gerada [4] e o ruído de fundo pode apresentar assinatura característica semelhante ao sinal. Portanto, a seleção de eventos em HEP é estimulante para a utilização de técnicas de aprendizado de máquina [5], que tem ganhado destaque em aplicações variadas, como rejeição de ruído de fundo [6] em experimentos de astropartículas e estudos de dinâmica de feixes de partículas [7] em aceleradores.
Estes aspectos podem ser encontrados no experimento ATLAS (A Toroidal LHC ApparatuS) [8] do acelerador de partículas LHC (Large Hadron Collider) [9] [10], instalado no CERN (Organização Europeia para Pesquisa Nuclear), Suíça. O ATLAS é o maior detector de partículas de propósito geral do LHC. É composto por diversos subsistemas de detecção, dentre eles o calorímetro com fina segmentação, com mais de 100.000 sensores responsáveis por medir a energia depositada pelo subproduto das colisões [11].

Elétrons são partículas de interesse destacado pelo ATLAS pois podem estar relacionadas a eventos físicos raros ainda não observados experimentalmente [12]. Um dos principais eventos de interesse são assinaturas relacionadas com o bóson $\mathrm{Z}$, que produz decaimento de dois elétrons $\left(Z \rightarrow e^{+} e^{-}\right)$, em que há forte interesse pelo experimento na detecção desse par combinado de assinaturas. Entretanto, jatos de natureza hadrônica produzidos nas colisões podem apresentar perfil de deposição de energia semelhante aos elétrons, o que dificulta o desempenho de classificação de assinaturas de interesse. Para lidar com essas restrições o ATLAS utiliza um sistema online de filtragem (trigger) dividido em duas etapas sequenciais. O primeiro nível de trigger (L1) é implementado em hardware e um segundo nível de filtragem, o trigger de alto nível - HLT (High Level Trigger) implementado em software.

Desde 2017 o ATLAS utiliza como algoritmo de identificação de elétrons no primeiro estágio do HLT (etapa rápida), um sistema de classificação baseado em um ensemble de redes neurais artificiais (NeuralRinger) [13] [14]. O ensemble foi alimentado por anéis topológicos que medem a energia em torno da célula mais energética [15]. O NeuralRinger foi desenvolvido com classificadores treinados em intervalos específicos de energia e diferentes regiões do detector. Esta estratégia permitiu que os classificadores neurais possam explorar as diferentes respostas do calorímetro dependendo da faixa de energia da partícula, bem como as distintas granularidades dos sensores em cada região do sistema de calorimetria [8].

No próximo período de tomada de dados agendado para 2021-2024, o LHC irá operar com $14 \mathrm{TeV}$ que é a máxima 
energia em colisões na qual foi projetado. O LHC realiza colisões de prótons em uma taxa de $40 \mathrm{MHz}$ e considerando que um evento medido pelo ATLAS tem $\approx 1,5$ MBytes, poderá ser gerado até $60 \mathrm{TBytes} / \mathrm{s}$ de informação a ser processada pelo experimento [16]. Espera-se ainda um aumento no número médio de interações por cruzamento de feixes, que pode dificultar a detecção da física de interesse com o possível empilhamento de sinais medidos no sistema de calorimetria (pile-up). Este aumento da ocupação do calorímetro distorce o padrão de deposição de energia das partículas, degradando a eficiência dos métodos de estimação de energia. Fato que compromete as medidas de calorimetria utilizadas pelo HLT. Este problema impacta o desempenho dos algoritmos de filtragem online, o que requer investigações para tornar os algoritmos de classificação do sistema de filtragem resilientes ao aumento do empilhamento de sinais [16].

Visando melhorias na filtragem online, o NeuralRinger vem sendo otimizado para atendimento às exigências do experimento. Uma das principais características adotadas para distinção de elétrons e jatos hadrônicos é o perfil lateral do chuveiro de partículas desenvolvido no calorímetro [17]. No trabalho [18], o perfil lateral do chuveiro foi usado em um mapeamento multidimensional de anéis topológicos em uma investigação primária para condições previstas para o LHC. A inclusão desta informação especializada em calorimetria (largura do chuveiro), permitiu a melhoria na detecção de elétrons e redução do ruído de fundo [18].

Este trabalho apresenta contribuições para a melhoria na detecção online de elétrons no ATLAS. O método proposto pondera o perfil dos anéis pela energia e distância do anel à região de interesse, incluindo a informação especialista da largura do chuveiro de partículas no sinais medidos. Os coeficientes exponenciais que ponderam a energia e a distância são ajustados iterativamente ao longo do treinamento de cada rede neural do ensemble. Este trabalho está dividido do seguinte modo: na seção 2 detalhes do experimento ATLAS e o sistema de filtragem online são apresentados. Na seção 3, a metodologia proposta é discutida. Os resultados encontrados com a metodologia proposta e comparações com o algoritmo de filtragem de referência são apresentados na seção 4 . Na seção 5 as conclusões e trabalhos futuros são discutidos.

\section{O EXPERIMENTO ATLAS}

O esquemático do experimento ATLAS $S^{1}$ é apresentado na Fig. 1. Com aproximadamente $45 \mathrm{~m}$ de comprimento e $25 \mathrm{~m}$ de altura o ATLAS, é composto por um detector de traço na região mais interna em torno do ponto de interação, rodeado por calorímetros e câmara de múons. $\mathrm{O}$ detector de traço está imerso em um campo magnético de $\approx 2 \mathrm{~T}$ produzido por

${ }^{1} \mathrm{O}$ ATLAS usa um sistema de coordenadas baseado na regra da mão direita, com sua origem no ponto de interação (PI) no centro do detector e no eixo $z$ ao longo do feixe. O eixo $x$ aponta do PI para o centro do anel do LHC e o eixo $y$ é perpendicular a este plano. Coordenadas cilíndricas $(r, \phi)$ são usadas no plano transverso, sendo $\phi$ o ângulo azimutal em torno do feixe. A pseudo-rapidez é definida em termos do ângulo polar $\theta$ (ângulo entre $x$ e $y$ ) como $\eta=-\ln [\tan (\theta / 2)]$. A distância angular $\Delta R$ é definida como $\Delta R=$ $\sqrt{(\Delta \eta)^{2}+(\Delta \phi)^{2}}$. um solenóide supercondutor e fornece reconstrução precisa de traços de partículas carregadas em uma faixa de pseudorapidez [8].

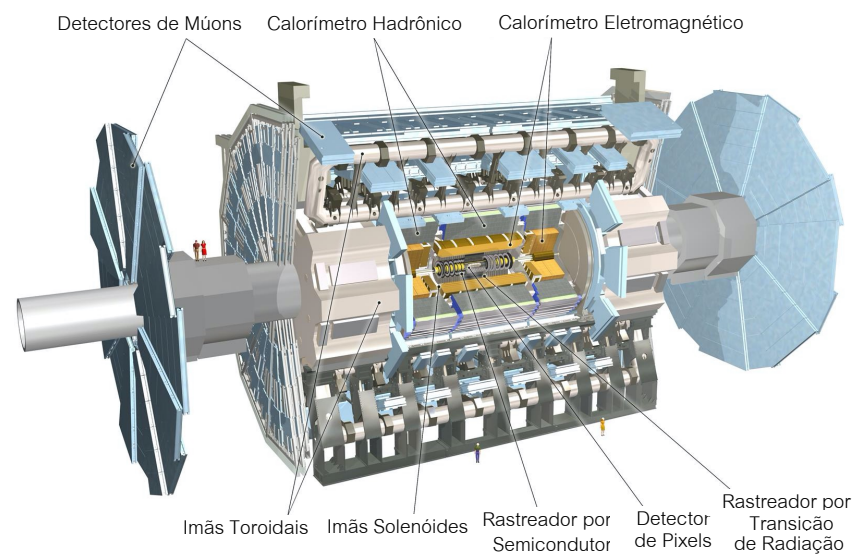

Figura 1: O experimento ATLAS e seus principais subsistemas de detecção. Adaptado de [8].

O sistema de calorimetria conforme indicado na Fig. 2 possui secções eletromagnéticas (EM) e hadrônicas (HAD). Ele é projetado para fornecer uma cobertura total em $\phi$ e cobre a faixa de pseudo-rapidez $|\eta|<4,9$, com granularidade fina sobre a região do detector interno. O calorímetro EM é um calorímetro de amostragem de chumbo/argônio líquido (Liquid-Argon - LAr) com geometria de acordeão [8].

O barril (região central do detector) é dividido em duas metades $(-1,475<\eta<0$ e $0<\eta<1,475)$ e dois componentes da extremidade $(1,375<|\eta|<3,2)$. A região de transição entre o barril e as tampas das extremidades $(1,37<|\eta|<1,52)$ contém material inativo adicional (crack) . Na região dedicada às medições precisas $(|\eta|<2,5)$ (excluindo o crack), o calorímetro EM é segmentado em três camadas longitudinais (EM1, EM2 e EM3) na direção da profundidade do chuveiro. Três camadas de calorímetro hadrônico circundam o calorímetro EM (HAD0, HAD1 e HAD2) utilizadas para medir energia de partículas com natureza hadrônica. $\mathrm{Na}$ frente do calorímetro acordeão, uma camada chamada préamostrador (PS) fina, cobrindo o intervalo de pseudo-rapidez $|\eta|<1,8$, é usada para corrigir a perda de energia das partículas antes de chegarem ao calorímetro.

\section{Trigger DE ElÉtrons no Detector ATLAS}

Um sistema de trigger, descrito na Fig. 3 projetado em dois níveis sequenciais é usado para filtrar candidatos a eventos de interesse no ATLAS [13]. O trigger de primeiro nível (L1) é implementado em hardware dedicado para reduzir a taxa de eventos da taxa de cruzamento de feixe de $40 \mathrm{MHz}$ para menos de $100 \mathrm{kHz}$. Esta etapa é limitada em 2,5 $\mu$ s para decidir quais eventos serão mantidos. O L1 também define as Regiões de Interesse (RoIs) que têm aglomerados de células do calorímetro (clusters) com alta $E_{T}$ ou traços de múon nas câmaras de múon. 


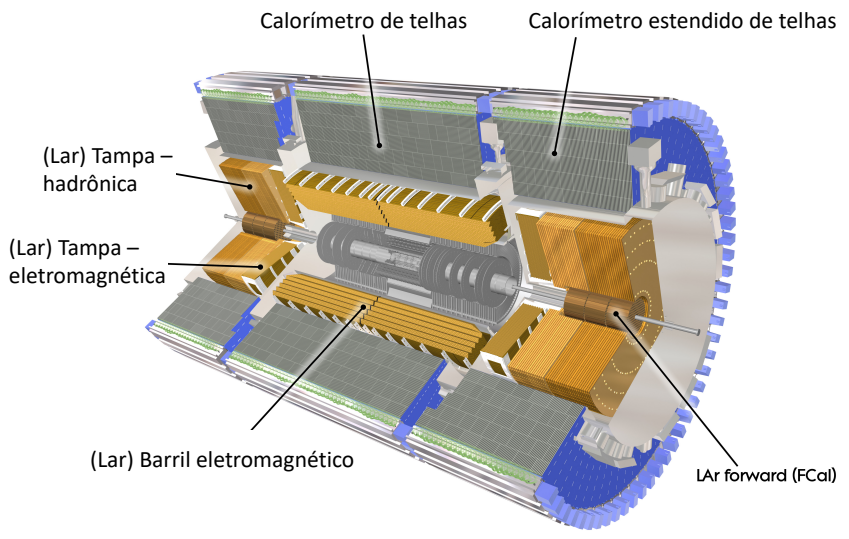

Figura 2: Esquemático detalhado dos calorímetros do ATLAS. Adaptado de [8].

Os eventos aceitos no L1 são processados pelo HLT, com base em algoritmos computacionais que reduzem o número de eventos a serem gravados em disco, a uma taxa média de cerca de $1,5 \mathrm{kHz}$. A seleção de eventos candidatos pelo HLT é realizada a cada etapa, de forma que se houver reprovação de um determinado evento em uma das etapas de trigger, as etapas subsequentes não serão executadas para este evento.

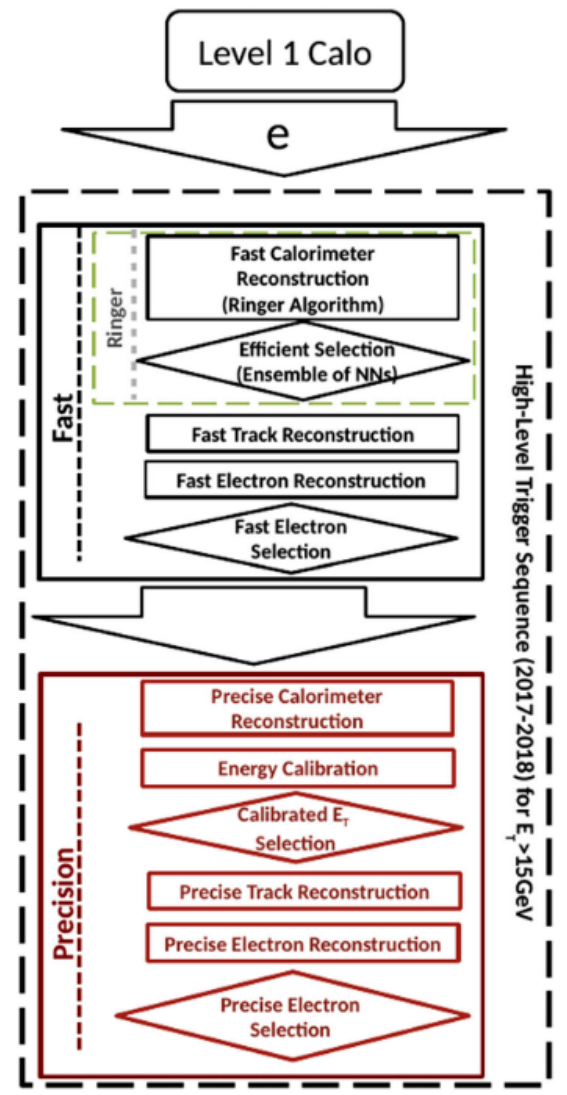

Figura 3: Diagrama simplificado do sistema de trigger empregado no ATLAS para elétrons na Run2 [13].
O HLT é subdividido nas etapas rápida e precisa. Na etapa rápida as RoIs selecionadas pelo L1 são analisadas. $\mathrm{O}$ algoritmo NeuralRinger usado como classificador na etapa rápida, realiza um mapeamento topológico em anéis concêntricos em torno da RoI (ver Fig. 4). O NeuralRinger, explora a geometria cônica do formato do chuveiro de partículas utilizando as células do calorímetro, para descrever a quantidade de energia depositada em cada anel concêntrico [19]. Os sinais em anéis alimentam um ensemble de redes neurais artificiais, para classificação do evento em sinal/ruído de fundo. O NeuralRinger permite a compactação da informação em 100 variáveis discriminantes (ver Tabela I), considerando uma quantidade de $\approx 1000$ sensores presentes na RoI.

\begin{tabular}{c|c|c|c|c|c|c|c}
\hline \hline Camadas & PS & EM1 & EM2 & EM3 & HAD0 & HAD1 & HAD2 \\
\hline Anéis & 8 & 64 & 8 & 8 & 4 & 4 & 4 \\
\hline \hline
\end{tabular}

Tabela I: Número de anéis concêntricos gerados em cada camada do calorímetro.

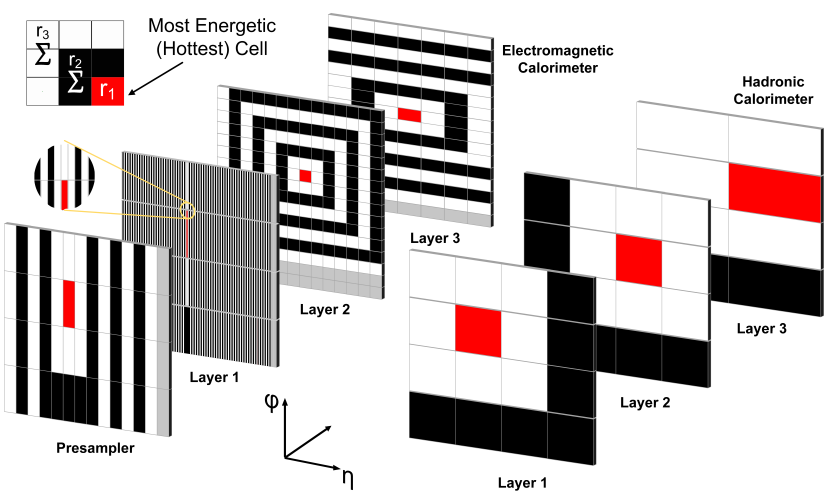

Figura 4: Representação dos anéis concêntricos gerados nas camadas do calorímetro do ATLAS pelo algoritmo anelador [13].

No calorímetro EM, os anéis são centralizados em torno da célula mais energética em cada camada, enquanto no calorímetro hadrônico a posição da célula mais energética na segunda camada do calorímetro EM é usada como eixo [20]. O NeuralRinger apresentou redução do falso alarme em até 3 vezes, na detecção de elétrons com energia acima de 15 $\mathrm{GeV}$ em relação ao algoritmo de seleção de eventos baseados em cortes rígidos em variáveis que descrevem o chuveiro de partículas (CutBased), que operou no HLT até 2017.

\section{A. Trabalhos Relacionados}

A proposta do mapeamento do perfil de deposição de energia em anéis concêntricos como sinais de entrada em redes neurais foi detalhada no trabalho [18]. A descrição do perfil de deposição de energia em anéis, alimentando uma rede neural completamente conectada encontrou $97 \%$ de eficiência para classificação de elétrons e 10,5\% de erro de classificação de jatos hadrônicos em eventos sem pile-up. Também em [18], a proposta do uso da largura do chuveiro como variável 
discriminante, para discriminação elétron/jato foi avaliada. A largura do chuveiro (variável $R_{p}$ ) unidimensional [21], pode ser definida conforme Eq. 1 .

$$
R_{p}=\frac{\sum_{i=1}^{C} E_{i}^{\alpha} r_{i}^{\beta}}{\sum_{i=1}^{C} E_{i}^{\alpha}},
$$

onde, $E_{i}$ representa a energia depositada em uma célula individual $i, r_{i}$ é a distância euclidiana entre o centro desta célula e a célula de energia máxima depositada, $\alpha$ e $\beta$ são parâmetros que pode ser ajustados e $C$ o número total de células. Usando a variável $R_{p}$ unidimensional como discriminante com corte linear, foi encontrado $97 \%$ de eficiência de classificação de elétrons, e $9 \%$ de jatos incorretamente classificados como elétrons. Estes resultados foram encontrados usando uma busca por otimização dos parâmetros $\alpha$ e $\beta$, para minimizar a norma do erro de classificação de jatos em taxas específicas de eficiência de detecção. O mapeamento $R_{p}$ multidimensional foi apresentado, usando a energia dos anéis topológicos (RpRing), conforme descrito na Eq. 2

$$
\begin{gathered}
R_{p}: X \rightarrow Y, \\
\left(E_{r i}, r d_{i}\right)=\frac{E_{r i}^{\alpha} r d_{i}^{\beta}}{\sum_{i=1}^{R} E_{i}^{\alpha}},
\end{gathered}
$$

em que, $r d_{i}$ representa o raio de cada anel medido em relação ao primeiro anel. Este método ofereceu a vantagem de combinar a compactação que a estrutura em anéis permite com o mapeamento discriminante $R_{p}$. Ainda em [18], uma rede neural foi adaptada no intuito de ser capaz de otimizar, durante o treinamento, os valores de $\alpha$ e $\beta$ aplicando retropropagação do erro até uma camada inicial, cujos neurônios foram responsáveis pelo mapeamento $R_{p}$. Utilizou-se como entrada os valores de energia e distância euclidiana de cada uma das células do espaço de entrada.

Neste trabalho é apresentada uma extensão da investigação realizada em [18]. Um sistema integrado foi desenvolvido usando o mapeamento $R_{p}$ multidimensional e um classificador neural. Os parâmetros $\alpha$ e $\beta$ foram incluídos em uma camada de entrada e integrados ao procedimento de treinamento do classificador. Utilizou-se como entrada os valores de energia dos anéis gerados pelo algoritmo anelador. Deste modo, os parâmetros que ponderam a energia do anel e sua distância em relação ao primeiro anel foram ajustados durante o treinamento dos classificadores. A seção IV] detalha a metodologia proposta.

\section{MÉtodo Proposto}

O fluxo de processamento proposto para uso do mapeamento $R_{p}$ nos anéis é indicado na Fig. 5. A energia dos anéis gerados nas células do calorímetro é preprocessada, conforme descrito na Eq.2. Os parâmetros $\alpha$ e $\beta$, são ajustados iterativamente ao longo do treinamento da rede neural, usando backpropagation, conforme indicado na Fig. 6

Para realizar o treinamento do sistema de classificação proposto, foram utilizados conjuntos de dados experimentais, coletados em colisões do LHC em 2017. O conjunto de dados

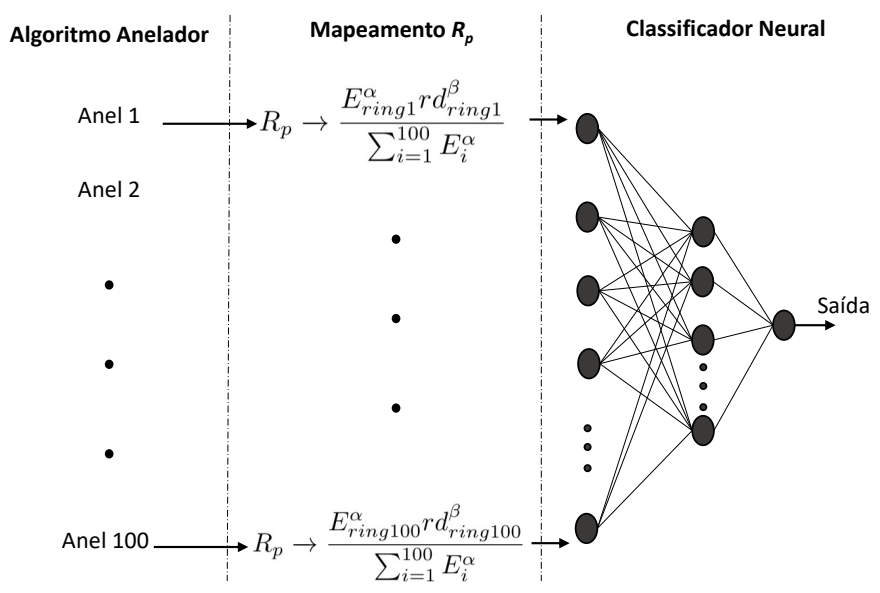

Figura 5: Fluxo de processamento do sistema de classificação desenvolvido. Os anéis concêntricos gerados pelo algoritmo anelador são processados pelo mapeamento $R_{p}$ e alimentam um classificador neural para discriminação elétron/jato.

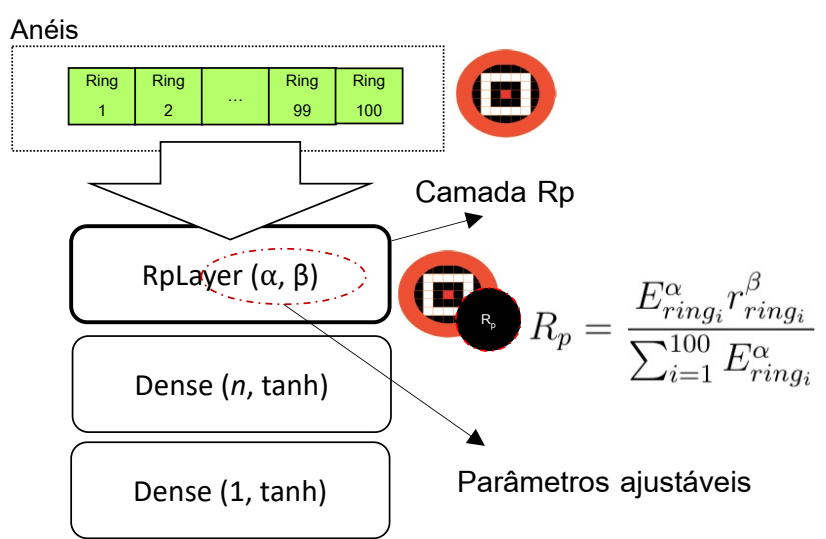

Figura 6: Diagrama da arquitetura da rede neural. Uma camada de entrada com parâmetros $\alpha$ e $\beta$ ajustáveis durante o treinamento é usada para realização do mapeamento $R_{p}$ multidimensional.

reservado para sinal é composto de assinaturas provenientes do decaimento do bóson $Z$ ( $Z \rightarrow e^{+} e^{-}$). Como os dados são de natureza experimental, critérios de decisão dos algoritmos de hipótese do ambiente offline são adotados para etiquetar assinaturas de sinal e ruído de fundo (usando modelos de física teórica).

Os conjuntos de dados selecionados para a classe de elétrons foram aqueles sinais aprovados como elétrons pelo método Tag and Probe (procedimento que utiliza aspectos de física teórica e medições em calorimetria para associar o par de elétrons que decaem do bóson Z) [22] usando o critério medium. O critério medium é um ponto de operação do algoritmo de classificação do offline, que permite desempenho equilibrado entre detecção de sinal $\left(P_{D}\right)$ e contaminação por ruído de fundo $\left(P_{F}\right)$. O conjunto de dados de ruído de fundo foi composto por eventos 
reprovados pelos algoritmos de reconstrução do offline usando o critério vloose. O critério vloose é um ponto de operação dos algoritmos de classificação do offline que permite elevada coleta de elétrons porém aumento na aceitação de ruído de fundo.

Os conjuntos de dados são separados por energia transversa $\left(E_{T}\right)$ e faixa em $\eta$ do sistema de calorimetria. Para cada faixa, descrita na Tab. [I] classificadores são treinados, formando um ensemble de 25 redes neurais. As Fig. 7 e 8 , apresentam a distribuição em $E_{T}$ e $\eta$ respectivamente de todos os anéis presentes no conjunto de dados utilizado.

Tabela II: Regiões em $\eta$ e faixas de energia empregadas para o ajuste dos modelos neurais.

\begin{tabular}{c|c|c|c|c|c}
\hline & \multicolumn{5}{|c}{ Intervalos } \\
\hline \hline $\mathbf{E}_{\mathbf{T}}(\mathrm{GeV})$ & {$[15,20]$} & {$[20,30]$} & {$[30,40]$} & {$[40,50]$} & {$[50, \infty[$} \\
\hline$\eta$ & {$[0 ; 0,80]$} & {$[0,80 ; 1,37]$} & {$[1,37 ; 1,54]$} & {$[1,54 ; 2,37]$} & {$[2,37 ; 2,50]$} \\
\hline
\end{tabular}

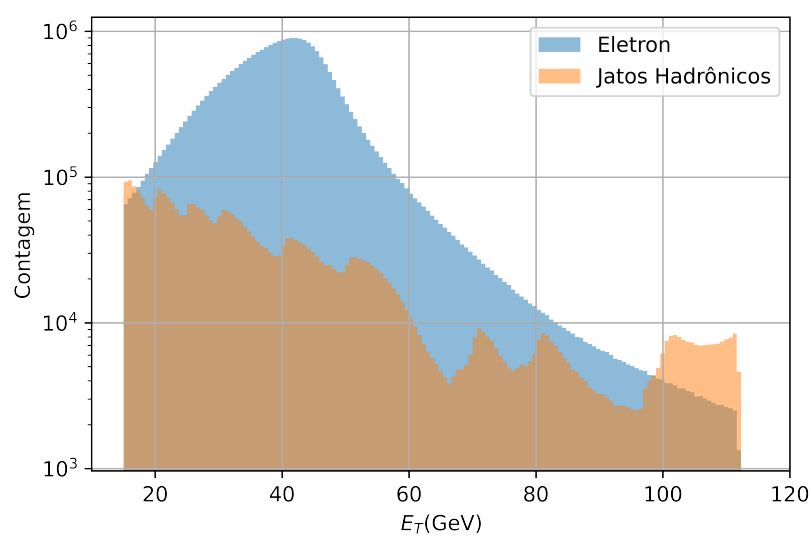

Figura 7: Distribuição em $E_{T}$ dos conjuntos de dados utilizados para treinamento do ensemble de redes neurais.

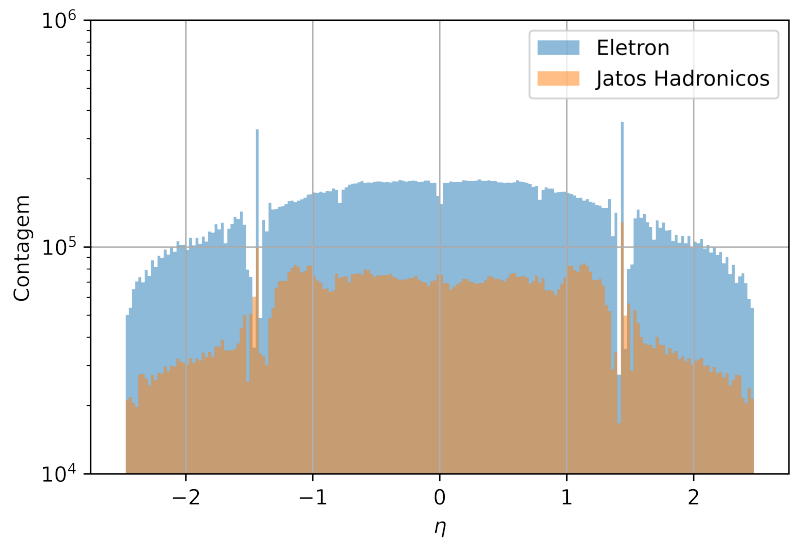

Figura 8: Distribuição pela coordenada $\eta$ do ATLAS dos conjuntos de dados utilizados para treinamento do ensemble.
A quantidade de neurônios na camada oculta foi escolhida, variando o número de neurônios entre 2 e 10. Para análise da flutuação estatística dos conjuntos de dados e seu impacto no treinamento dos modelos, adotou-se um método de validação cruzada (Stratified $k$-fold) que realiza 10 sorteios, para separação dos conjuntos para as fases de treinamento e teste com as proporções de $90 \%$ e $10 \%$, respectivamente. Para cada sorteio realizado, 10 inicializações de treinamento são efetuadas para analisar a possível ocorrência de mínimos locais. Dois critérios de parada do treinamento foram adotados. $\mathrm{O}$ treinamento dos modelos é interrompido quando 25 sucessivas falhas de melhoria do índice soma-produto (SP) (conforme Eq. 3) ocorre, ou quando o número máximo de 5000 épocas de treinamento é alcançado. O índice SP é um indicador de equilíbrio entre a probabilidade de detecção de sinal $\left(P_{D}\right)$ e a taxa de falso alarme $\left(P_{F}\right)$ [23] [24].

$$
S P=\sqrt{\sqrt{P_{D}\left(1-P_{F}\right)} \times \frac{P_{D}+\left(1-P_{F}\right)}{2}},
$$

em que $P_{D}$, é a medida percentual de eventos da classe de sinal, classificados como sinal. A probabilidade de falso alarme $\left(P_{F}\right)$ é a medida percentual de eventos de ruído de fundo classificados como sinal.

Após o treinamento, os classificadores com maior índice SP são selecionados para realização do ajuste do limiar de decisão, em função do número médio de interações por cruzamento de feixes $(\langle\mu\rangle)$. Este procedimento visa verificar a influência do pile-up na taxa de falso alarme, para um mesmo ponto de operação de probabilidade de detecção. Também tem o propósito de manter a eficiência de detecção $\left(P_{D}\right)$ de sinais constante, independente da taxa de empilhamento. Manter a taxa de $P_{D}$ constante mesmo com as variações da taxa de empilhamento é muito importante para não deteriorar a eficiência geral do HLT (saída da etapa precisa). Para esta correção, a função de ativação tangente hiperbólica do neurônio de saída é retirada, e substituída por uma função linear, em que os coeficientes $a$ e $b$ (ver Eq. 4) são ajustados para manter a probabilidade de detecção semelhante a referência (taxa de $P_{D}$ do NeuralRinger) em função de $\langle\mu\rangle$.

$$
\delta(\langle\mu\rangle)=a\langle\mu\rangle+b,
$$

em que $a$ e $b$ são os coeficientes da curva.

Após o treinamento, o desempenho obtido (usando os subconjuntos sorteados para teste) pelo método RpRing foi comparado com o NeuralRinger e com o CutBased ${ }^{2}$ Foram destacados na seção $\mathrm{V}$ resultados obtidos na faixa de energia $[30 ; 40] \mathrm{GeV}$ e $\eta[0 ; 0,8]$. O sumário das principais configurações do treinamento dos modelos é apresentado na Tabela 【II.

\section{REsultados}

Inicialmente avaliou-se a quantidade de neurônios na camada oculta, e o seu impacto nas medidas de avaliação de

\footnotetext{
${ }^{2}$ Método de classificação baseado em cortes lineares em variáveis de formato do chuveiro de partículas, que operou no HLT do ATLAS até 2017.
} 
Tabela III: Resumo dos principais parâmetros utilizados no treinamento dos classificadores.

\begin{tabular}{c|c}
\hline \hline Algoritmo de treinamento & ADAM \\
\hline Taxa de Aprendizado & 0.010 \\
\hline Alvo de Elétron/Ruído de Fundo & $+1 /-1$ \\
\hline Critério de Parada & 25 sucessivas falhas de melhorias em SP \\
\hline Número de Épocas & $10(9$ para treino, 1 para teste $)$ \\
\hline Número de Subconjuntos & 0.0001 \\
\hline Goal Fitness Train & 5000 \\
\hline Épocas & 1 \\
\hline Número de Camadas Ocultas & Tangente Hiperbólica \\
\hline Função de ativação & Completamente conectada \\
\hline Arquitetura &
\end{tabular}

desempenho. A Fig 9 apresenta o boxplot dos índices SP encontrados (na faixa de energia $E_{T}$ : $[30,40] \mathrm{GeV}$ e $\eta$ : [0, $0,80]$ ), para arquiteturas avaliadas entre 2 e 10 neurônios na camada oculta. Observa-se que a partir de 7 neurônios, não há significativa mudança na distribuição dos valores de SP encontrados.

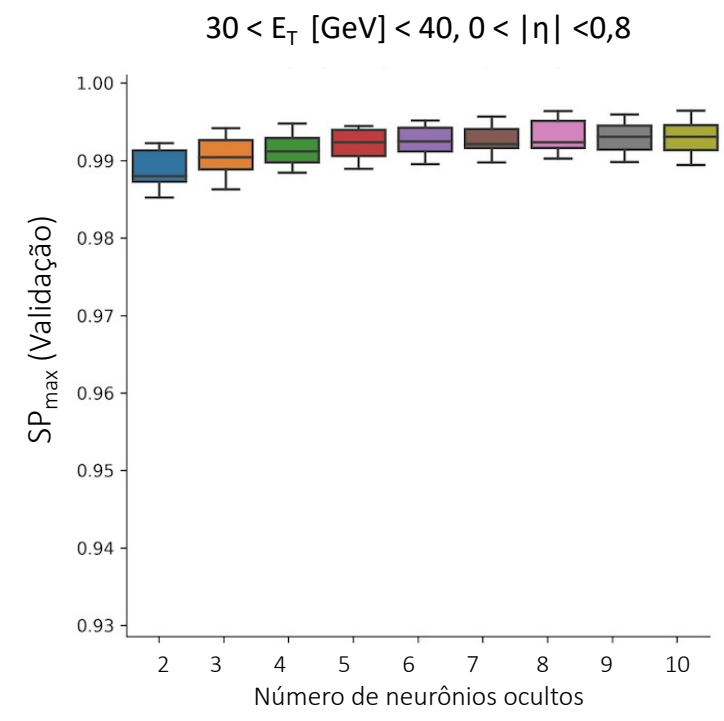

Figura 9: Boxplot dos valores encontrados para o índice SP com o conjunto de teste, para os modelos treinados com 2 a 10 neurônios na camada oculta.

As Tabelas IV e $\mathrm{V}$ apresentam os índices de probabilidade de detecção $\left(P_{D}\right)$ e probabilidade de falso alarme $\left(P_{F}\right)$ para os pontos de operação tight (o critério tight é o ponto de operação do classificador que indica que aquela partícula possui uma alta probabilidade de ser um elétron) e vloose dos sistemas de classificação respectivamente. Estas tabelas indicam os resultados de eficiência geral (considerando os resultados de todos os classificadores do ensemble). Os resultados obtidos indicam que o método RpRing com 7 neurônios proporciona uma redução na taxa de falso alarme de 0.54 pontos percentuais quando comparado a referência (NeuralRinger), operando no ponto de operação tight. Quando o ponto de operação é ajustado para vloose, o RpRing encontra uma redução no falso alarme em 1.37 pontos percentuais, se comparado a referência.

Tabela IV: Eficiência geral usando validação cruzada para cada técnica de classificação para a operação Tight.

\begin{tabular}{c|c|c}
\hline \hline & $P_{D}[\%]$ & $P_{F}[\%]$ \\
\hline CutBased & 99.05 & 17.63 \\
\hline NeuralRinger (Referência) & $99.05 \pm 0.00$ & $1.33 \pm 0.05$ \\
\hline RpRing (mlp2) & $99.04 \pm 0.00$ & $1.00 \pm 0.01$ \\
\hline RpRing (mlp3) & $99.05 \pm 0.01$ & $0.94 \pm 0.01$ \\
\hline RpRing (mlp4) & $99.05 \pm 0.00$ & $0.90 \pm 0.01$ \\
\hline RpRing (mlp5) & $99.04 \pm 0.00$ & $0.86 \pm 0.01$ \\
\hline RpRing (mlp6) & $99.05 \pm 0.00$ & $0.84 \pm 0.01$ \\
\hline RpRing (mlp7) & $\mathbf{9 9 . 0 5} \pm \mathbf{0 . 0 1}$ & $\mathbf{0 . 7 9} \pm \mathbf{0 . 0 1}$ \\
\hline RpRing (mlp8) & $99.05 \pm 0.01$ & $0.79 \pm 0.01$ \\
\hline RpRing (mlp9) & $99.05 \pm 0.00$ & $0.80 \pm 0.01$ \\
\hline RpRing (mlp10) & $99.05 \pm 0.01$ & $0.80 \pm 0.01$ \\
\hline \hline
\end{tabular}

Tabela V: Eficiência geral usando validação cruzada para cada técnica de classificação Very Loose.

\begin{tabular}{c|c|c}
\hline \hline & $P_{D}[\%]$ & $P_{F}[\%]$ \\
\hline CutBased & 99.65 & 32.67 \\
\hline NeuralRinger (Referência) & $99.67 \pm 0.00$ & $2.86 \pm 0.17$ \\
\hline RpRing (mlp2) & $99.66 \pm 0.00$ & $2.26 \pm 0.44$ \\
\hline RpRing (mlp3) & $99.67 \pm 0.01$ & $2.73 \pm 1.70$ \\
\hline RpRing (mlp4) & $99.67 \pm 0.00$ & $1.68 \pm 0.02$ \\
\hline RpRing (mlp5) & $99.67 \pm 0.00$ & $1.59 \pm 0.04$ \\
\hline RpRing (mlp6) & $99.67 \pm 0.00$ & $1.55 \pm 0.03$ \\
\hline RpRing (mlp7) & $\mathbf{9 9 . 6 7} \pm \mathbf{0 . 0 1}$ & $\mathbf{1 . 4 9} \pm \mathbf{0 . 0 1}$ \\
\hline RpRing (mlp8) & $99.67 \pm 0.01$ & $1.49 \pm 0.01$ \\
\hline RpRing (mlp9) & $99.67 \pm 0.00$ & $1.51 \pm 0.01$ \\
\hline RpRing (mlp10) & $99.66 \pm 0.01$ & $1.52 \pm 0.01$ \\
\hline \hline
\end{tabular}

Durante o treinamento das redes neurais uma monitoração foi realizada para verificar possível incidência de overfitting. A Fig. 10 apresenta como exemplo, duas curvas de treinamento para o modelo neural que obteve melhor desempenho em SP na faixa de energia $[30 ; 40] \mathrm{GeV}$ e $\eta[0 ; 0,80]$. Em 10 (a) é apresentado a evolução do erro médio quadrático ao longo das épocas de treinamento indicando que, neste caso, o erro sobre o conjunto de dados de validação não apresenta redução para um número de épocas acima de $\approx 175$. Em 10 (b) o comportamento do índice SP ao longo das épocas de treinamento é apresentado, em que por meio da estratégia de parada antecipada, o treinamento foi interrompido após 25 épocas sem melhorias em relação ao índice SP.

A evolução dos parâmetros $\alpha$ e $\beta$ para o classificador neural selecionado que obteve melhor desempenho em SP na faixa de energia [30;40] GeV e $\eta[0 ; 0,80]$ é apresentada na Fig. 11

As Fig. 12 e 13 apresentam os histogramas de saída da rede neural selecionada, que obteve melhor desempenho em SP na faixa de energia $[30 ; 40] \mathrm{GeV}$ e $\eta[0 ; 0,80]$ para sinal e ruído de fundo respectivamente. A função de ativação não linear foi removida do neurônio de saída e uma curva linear foi ajustada em função do nível de empilhamento $(\langle\mu\rangle)$. A curva foi ajustada para manter o $P_{D}$ do classificador RingerRp com o mesmo desempenho do classificador de referencia (NeuralRinger). Cada ponto da curva (pontos azuis) indica um 


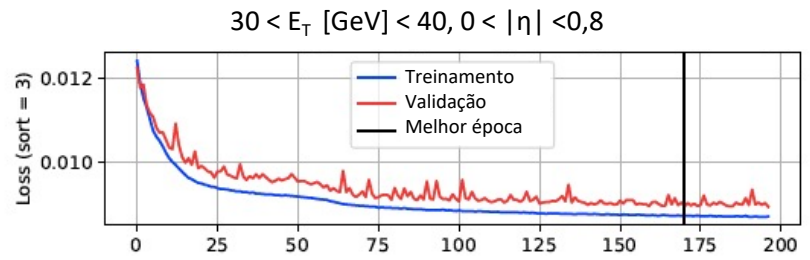

(a)

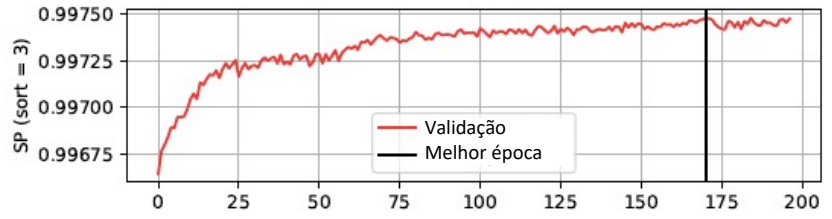

(b)

Figura 10: Curvas de monitoramento de treinamento para o classificador neural treinado no intervalo de $E_{T}[30 ; 40] \mathrm{GeV}$ e $\eta[0 ; 0,80]$. Em (a) a curva do erro médio quadrático é apresentada e em (b) a curva do índice SP para cada época de treinamento é mostrada.

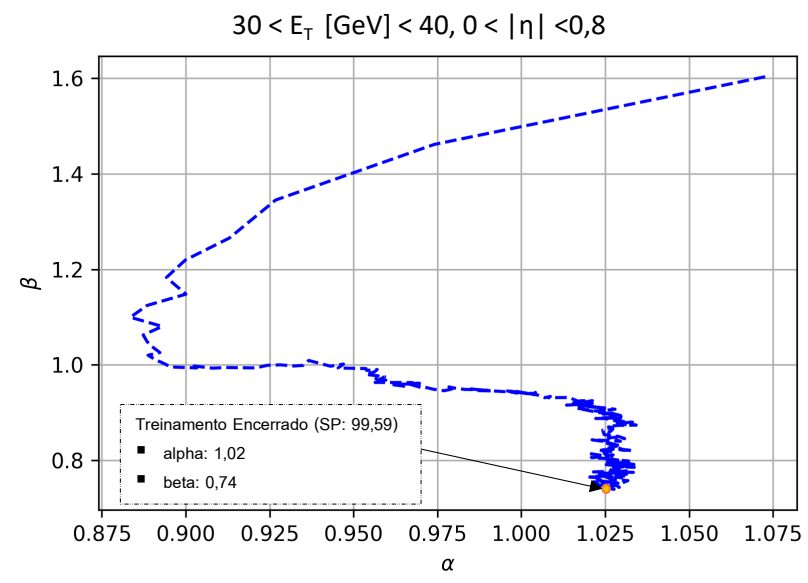

Figura 11: Variação dos parâmetros $\alpha$ e $\beta$ ao longo das épocas de treinamento.

limiar de decisão que mantém o $P_{D}$ de $99.18 \%$ considerando a variação do empilhamento. Na Fig. 12 observa-se que a maior quantidade de sinais está posicionada a direita da reta que foi ajustada pela intensidade de empilhamento, indicando que a maioria dos sinais foram corretamente discriminados pelo classificador.

Observa-se na Fig. 13 que a maioria das assinaturas de jatos hadrônicos estão a esquerda da reta ajustada em função do empilhamento, indicando que a maior parte destes eventos foram corretamente discriminados pelo RpRing.

\section{CONCLUSÕES}

O experimento ATLAS é o maior detector de partículas de proposito geral do LHC, contribuindo significativamente para diversas descobertas científicas. Devido ao intenso ruído de fundo produzido nas colisões e uma elevada quantidade de

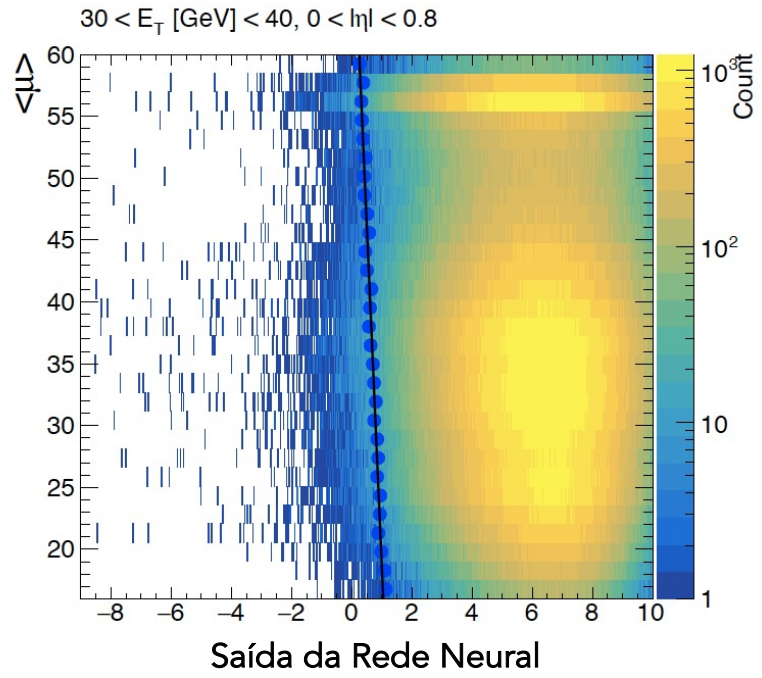

Figura 12: Histograma de saída da rede neural, com exemplos de sinal (elétrons), selecionada com melhor desempenho em SP na faixa de energia $[30 ; 40] \mathrm{GeV}$ e $\eta[0 ; 0,80]$.

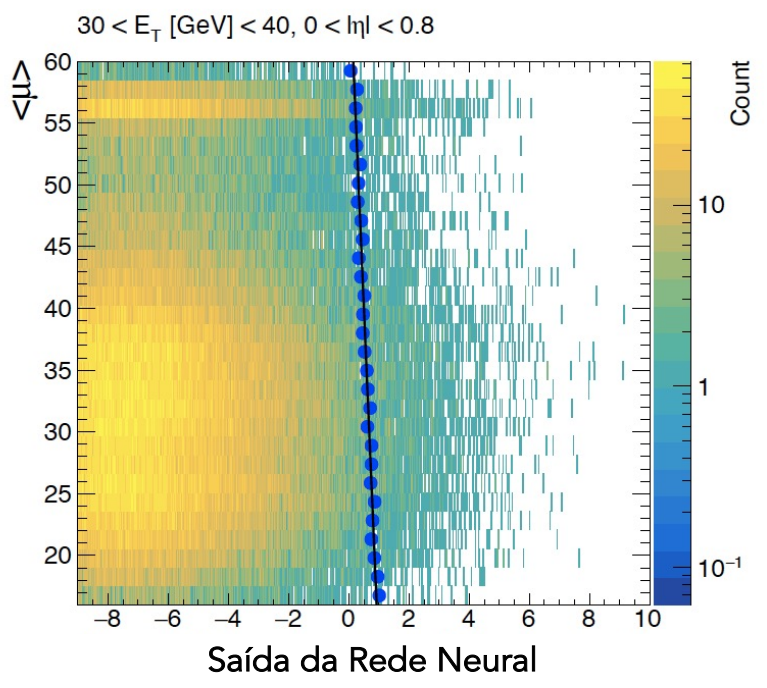

Figura 13: Histograma de saída da rede neural, com exemplos de ruído de fundo (jatos), selecionada com melhor desempenho em SP na faixa de energia $[30 ; 40] \mathrm{GeV}$ e $\eta[0 ; 0,80]$.

canais de leitura, um sistema rápido e eficiente de filtragem online é necessário. $\mathrm{O}$ algoritmo NeuralRinger, baseado em um ensemble de redes neurais artificiais, opera no sistema de filtragem online do ATLAS desde 2017, e a investigação de sua otimização vem sendo realizada, para atendimento das exigências do novo período de tomada de dados. Neste trabalho avaliou-se o uso da informação especialista do perfil lateral do chuveiro de partículas ( $R_{p}$ multidimensional), junto aos anéis gerados gerados pelo NeuralRinger. Os resultados 
da inclusão desta característica nos sinais formatados em anéis indicaram uma redução na taxa de falso alarme de 0,54 e 1,37 pontos percentuais para os pontos de operação Tight e Very Loose respectivamente (ambas configurações com 7 neurônios na camada oculta), se comparado ao NeuralRinger usando dados experimentais de 2017. Os resultados encontrados motivam a exploração da metodologia proposta e para trabalhos futuros, dados de colisão de 2018 podem ser utilizados para verificar o impacto da técnica proposta. Redes com arquiteturas convolucionais também podem ser avaliadas.

\section{AGRADECIMENTOS}

Os autores agradecem a FAPESB, CNPq, FAPERJ e RENAFAE pelo apoio financeiro. $\mathrm{O}$ presente trabalho foi realizado com apoio da Coordenação de Aperfeiçoamento de Pessoal de Nível Superior - Brasil (CAPES) - Código de Financiamento 001.

\section{REFERÊNCIAS}

[1] J. Fernández-Tejero, V. Fadeyev, C. Fleta, J. Hacker, M. Ullán, and Y. Unno, "Microelectronic test structures for the development of a strip sensor technology for high energy physics experiments," Nuclear Instruments and Methods in Physics Research Section A: Accelerators, Spectrometers, Detectors and Associated Equipment, vol. 969, p. 163971, 2020.

[2] T. Boccali, "Computing models in high energy physics," Reviews in Physics, vol. 4, p. 100034, 2019. [Online]. Available: https: //www.sciencedirect.com/science/article/pii/S2405428319300449

[3] N. Nottbeck, D. C. Schmitt, and P. D. V. Büscher, "Implementation of high-performance, sub-microsecond deep neural networks on FPGAs for trigger applications," Journal of Instrumentation, vol. 14, no. 09, pp. P09 014-P09 014, sep 2019.

[4] D. Sarkar, M. P., P. S., N. Chouhan, C. Borwankar, A. Bhattacharya, A. Tickoo, and R. Rannot, "Comparison of data storage and analysis throughput in the light of high energy physics experiment mace," Astronomy and Computing, vol. 33, p. 100409, 2020.

[5] S. Benson and K. Gizdov, "Nndrone: A toolkit for the mass application of machine learning in High Energy Physics," Computer Physics Communications, vol. 240, pp. 15-20, 2019.

[6] S. Spencer, T. Armstrong, J. Watson, S. Mangano, Y. Renier, and G. Cotter, "Deep learning with photosensor timing information as a background rejection method for the cherenkov telescope array," Astroparticle Physics, vol. 129, p. 102579, 2021.

[7] P. Arpaia, G. Azzopardi, F. Blanc, G. Bregliozzi, X. Buffat, L. Coyle, E. Fol, F. Giordano, M. Giovannozzi, T. Pieloni, R. Prevete, S. Redaelli, B. Salvachua, B. Salvant, M. Schenk, M. S. Camillocci, R. Tomás, G. Valentino, F. Van der Veken, and J. Wenninger, "Machine learning for beam dynamics studies at the cern large hadron collider," Nuclear Instruments and Methods in Physics Research Section A: Accelerators, Spectrometers, Detectors and Associated Equipment, vol. 985, p. 164652, 2021

[8] A. Collaboration, "The ATLAS experiment at the CERN Large Hadron Collider," Journal of Instrumentation, vol. 3, no. 08, pp. S08 003-S08 003, aug 2008. [Online]. Available: https://doi.org/10. 1088/1748-0221/3/08/s08003

[9] L. Evans and P. Bryant, "LHC machine," Journal of Instrumentation, vol. 3, no. 08, pp. S08 001-S08 001, aug 2008.

[10] O. Brüning, H. Burkhardt, and S. Myers, "The Large Hadron Collider," Progress in Particle and Nuclear Physics, vol. 67, no. 3, pp. 705-734, 2012.

[11] R. Wigmans, Calorimetry: Energy Measurement in Particle Physics, ser. International series of monographs on physics. Clarendon Press, 2017.

[12] A. Collaboration, "Evidence for Higgs boson decays to a low-mass dilepton system and a photon in pp collisions at $\sqrt{s}=13 \mathrm{TeV}$ with the ATLAS detector," Physics Letters B, vol. 819, p. 136412, 2021.

[13] G. Aad, Abbott et al., "Performance of electron and photon triggers in ATLAS during lhe run 2," Eur. Phys. J. C, vol. 80, p. 47. 56 p, Sep 2019.
[14] "An Ensemble of Neural Networks for Online Filtering Implemented in the ATLAS Trigger System," CERN, Geneva, Tech. Rep., Jul 2018. [Online]. Available: https://cds.cern.ch/record/2632911

[15] J. V. Da Fonseca Pinto, "Ring-shaped Calorimetry Information for a Neural EGamma Identification with ATLAS Detector," CERN, Geneva, Tech. Rep., Mar 2016. [Online]. Available: https://cds.cern.ch/record/ 2142761

[16] A. Collaboration, "Projected ATLAS electron and photon trigger performance in run 3," Proceedings of Science, vol. ICHEP2020, p. 757 , 2021.

[17] D. Acosta, S. Buontempo, L. Calôba, R. DeSalvo, A. Ereditato, R. Ferrari, G. Fumagalli, G. Goggi, W. Hao, A. Henriques, L. Linssen, M. Liyan, A. Maio, M. Mondardini, B. Ong, H. Paar, F. Pastore, E. Pennacchio, L. Poggioli, G. Polesello, F. Riccardi, A. Rimoldi, C. Scheel, J. Seixas, A. Simon, M. Sivertz, P. Sonderegger, M. Souza, Z. Thomé, V. Vercesi, Y. Wang, R. Wigmans, and C. Xu, "Lateral shower profiles in a lead/scintillating fiber calorimeter," Nuclear Instruments and Methods in Physics Research Section A: Accelerators, Spectrometers, Detectors and Associated Equipment, vol. 316, no. 2, pp. 184-201, 1992.

[18] J. Seixas, L. Caloba, M. Souza, A. Braga, and A. Rodrigues, "Neural second-level trigger system based on calorimetry," Computer Physics Communications, vol. 95, no. 2, pp. 143-157, 1996.

[19] T. Ciodaro, D. Deva, J. de Seixas, and D. Damazio, "Online Particle Detection by Neural Networks Based on Topologic Calorimetry Information," 14th International Workshop On Advanced Computing And Analysis Techniques In Physics Research, Uxbridge, West London, UK, 5 - 9 Sep 2011, Sep 2011. [Online]. Available: http://cds.cern.ch/record/1379508

[20] W. Spolidoro Freund and A. Collaboration, "An Ensemble of Neural Networks for Online Electron Filtering at the ATLAS Experiment," CERN, Geneva, Tech. Rep., May 2019. [Online]. Available: https://cds.cern.ch/record/2675025

[21] C. V. Scheel, The spaghetti calorimeter. Research, development, application. Amsterdam (Netherlands): International Atomic Energy Agency, 1994.

[22] A. Collaboration, "Electron efficiency measurements with the ATLAS detector using 2012 lhc proton-proton collision data," Eur. Phys. J. C, vol. 195 , no. 77 , pp. 1-45, 2017.

[23] R. C. Torres, D. E. F. de Lima, E. F. de Simas Filho, and J. M. de Seixas, "Neural online filtering based on preprocessed calorimeter data," in 2009 IEEE Nuclear Science Symposium Conference Record (NSS/MIC), 2009, pp. 530-536.

[24] E. F. Simas Filho, J. M. de Seixas, and L. P. Calôba, "Modified postnonlinear ica model for online neural discrimination," Neurocomputing, vol. 73, no. 16, pp. 2820-2828, 2010, 10th Brazilian Symposium on Neural Networks (SBRN2008). [Online]. Available: https://www. sciencedirect.com/science/article/pii/S0925231210002511 